\title{
ARCHIVAL LIFE EXPECTANCY OF 3M MAGNETO-OPTIC MEDIA
}

\author{
William P. Murray \\ 3M Optical Recording \\ 544-2N-01 3M Center \\ Saint Paul, MN 55144
}

\begin{abstract}
This work developed a model of life expectancies such that estimates could be made under a variety of temperatures and relative humidities. The model was such that a confidence interval could be established for any given amount of product compliance, at any temperature and relative humidity within a realistic storage range. A modified Eyring model was chosen to evaluate the growth kinetics of Byte Error Rates [bytes with errors + total bytes tested]. This model was then applied to a matrix of accelerated test data and fit to a variety of failure time distributions. The best fit was selected and estimates calculated at a variety of conditions. The confidence interval of these estimates were also made along with the percent of the population complying with these estimates.

KEYWORDS: Reliability, Eyring, Lognormal, Life Expectancy, Byte Error Rate,
\end{abstract}

\section{INTRODUCTION}

Today's options for data storage media are widely diverse and new variations are continually being introduced. Even within one technology, such as magneto-optic media, there are still many choices to be made. Different sizes, formats, data densities, rates and constructions are already on the market and new versions are being introduced at an increasing frequency. Along with this is the trend toward increased reliance on electronic data storage, increased complexity and importance of the data stored and increased requirements for the length of time that data must be stored.

The factors that affect the suitability of a particular storage format include many parameters such as total system cost and cost-per-byte, capacity and expandability, access rates, data security, compatibility and transportability. These factors are easily defined and measured.

The life-expectancy of the media is much more difficult to define and, realistically, impossible to measure. Compared to early experimental versions, the magneto-optic media of today offers a higher degree of stability to the effects of aging. Advances in raw materials, thin films, cleanliness and manufacture have led to products with life expectancies of several decades. This may be very comforting to some but to those that require accurate and precise life estimates at usage stresses, a better answer is required.

Historically, the approach to obtaining accelerated life estimates have been based on the Arrhenius relationship of reaction rates as a function of temperature. The equation written in its customary form:

$$
k=A e^{-(E / R T)}
$$

$$
\text { where: } \begin{aligned}
& k=\text { rate parameter } \\
& A=\text { pre-exponential factor } \\
& E=\text { activation energy } \\
R & =\text { universal gas constant } \\
T & =\text { temperature }
\end{aligned}
$$

was empeirically derived. Its ease of use accounts for its widespread appearance in accelerated testing literature. It is, however, of very limited use and is unacceptable for the requirements of this application. The principal drawback is that it allows life estimates to be made only as a function of one variable, temperature. As we will concern ourselves with developing a model for both temperature and relative humidity, a more comprehensive relationship must be utilized.

$$
\text { The Eyring Model, }
$$

$$
k=A T^{a} e^{-(E / R T)} e^{-(B+C / T) R H}
$$

is an expression that was derived from the laws of thermodynamics [1]. It may be expanded to include stresses other than temperature and relative humidity if desired. This acceleration model easily fits the requirements for this work.

In addition to the acceleration model, one must know the distribution of the estimated failure times. The most commonly known distribution is the "normal" distribution. This distribution was derived by Karl Gauss to model the distri- 
bution of measurement errors in astronomy. It's application is appropriate to systems where each of a rather large number of random variables makes a rather small and independent contribution to the overall effect [2]. This distribution produces the familiar Gaussian or bell-shaped curve.

Familiarity with a particular distribution is not sufficient reason to justify it's application to failure rate data. Both the Weibull and the lognormal distribution may, and quite likely will, be better suited to end-of-life(EOL) distributions of $M$ O media. The Weibull distribution finds applications where the EOL is brought about by the failure of the weakest element of the test specimen [3]. Such would be the case if EOL were caused by contaminants or flaws in the disk.

Application of the lognormal distribution is appropriate if there are several independent and multiplicative factors which influence the life expectancy according to the magnitude of the factor and the age of the specimen when the factor was introduced. The lognormal distribution may be the preferred model when failure is due to chemically induced corrosion influenced by variables such as $\mathrm{pH}$ or diffusion [4].

There are several other distributions which also may be applied to life expectancy models. There is no single distribution which is the best for all applications. One must fit the data to several different models, and utilize the best fitting and technically justifiable method.

With a sound basis for relating rates to both temperatures and relative humidities plus a failure distribution based on the product's behavior, one can then make meaningful estimates of life expectancies at a variety of conditions. It will then be possible for the user to ascertain data life under the realistic archival storage conditions rather then somewhat extreme relative humidity conditions chosen for the convenience of the experimenter.

\section{EXPERIMENTAL}

Ten sets of disks were selected randomly from the $3 \mathrm{M}$ standard process. A set consisted of 16 sides. Each side was constructed as follows: A polycarbonate substrate was coated by a silicon carbide dielectric. AnFeTbCo rare earthtransition metal (RE-TM) alloy with a Ta corrosion inhibitor was coated next, followed by a second silicon carbide dielectric. The reflector layer was coated next and then finally covered with an organic sealcoat. The two individual sides were bonded together with a thermoplastic adhesive.
The disk was then placed in a plastic cartridge.

The condition of each disk at the beginning-of-life was measured for BER by writing a pseudo-random pattern over 19 evenly spaced bands of 50 tracks per 1000 . The bands started at track 500 and continued to track 18549. This pattern was then tested for errors with Error Correction Code (ECC) turned off. Throughout the entire testing duration, the same identical ISO standard M-O drive was used for all BER measurements.

Each set of disks was then exposed to one of ten accelerated aging cells. Each cell was set to reach a different temperature/relative humidity combination for a preset interval of time. The rate of change of these variables in going from ambient conditions, to stress condition and back to ambient was under program control. Table 1 shows the conditions used for this experiment.

Table 1: Experimental conditions by cell number

$\begin{array}{cccccc}\text { Cell } & \text { T }^{\circ} \mathrm{C} & \text { \%RH } & \text { Cell* } & \text { T }^{\circ} \mathrm{C} & \text { \%RH } \\ 1 & 80 & 85 & 6 & 70 & 85 \\ 2 & 80 & 75 & 7 & 70 & 75 \\ 3 & 80 & 65 & 8 & 60 & 85 \\ 4 & 80 & 55 & 9 & 60 & 65 \\ 5 & 80 & 3 & 10 & 50 & 85\end{array}$

The stress intervals varied inversely with the temperature. The $80^{\circ} \mathrm{C}$ cells used a 500 hour interval, the $75^{\circ} \mathrm{C}$ cells used a 750 hour interval and the $60^{\circ} \mathrm{C}$ and $50^{\circ} \mathrm{C}$ cells used a 1000 hour interval. Four stress intervals were used for each cell which, combined with the zero hour test, produced five points per disk for evaluating the rate of change. At the end of each accelerated aging interval, each disk was measured for BER by read-only testing of the initial data.

\section{Calculations}

The change of BER versus time was determined by regressing the BER for each disk versus the time exposed in the accelerated aging cell. The maximum likelihood estimate (MLE) was used for all regressions in this report. This technique was chosen, a priori, due to its ability to handle life expectancy data. The MLE can be used where there is censored data, non-normal error distributions and interval failure times. In cases where error terms are normal, there 
is no censored data and only exact times to failure, then the results are identical to least squares linear regression.

It has been shown historically that the logarithmic change of BER is a linear function of time [5]. Graphic analysis of this work is in agreement. The MLE was therefore made using $\log$ values. Table 2 shows the BER data for each disk and each interval in cell 1. Data was collected and analyzed in a similar manner for each of the other nine cells in this experiment.

Table 2: BER $\times E^{-5}$ of disks exposed in cell 1

\begin{tabular}{|c|c|c|c|c|c|}
\hline DISK II & OघRS & SWO KRE & $1000 \mathrm{HRS}$ & 1500HDS & 2000मHRS \\
\hline FAB & 621 & 768 & 1800 & 7.270 & 1710 \\
\hline ALEAB & 624 & 660 & 733 & 1.010 & $1: 840$ \\
\hline PLB-27A & 526 & 562 & .630 & 841 & 562 \\
\hline PLE-2TB & .144 & 542 & 573 & $\overline{815}$ & .903 \\
\hline 7271A & 1330 & 1840 & 1450 & 1590 & 1850 \\
\hline THSIB & 814 & 156 & 146 & 605 & 135: \\
\hline PLE-75A & .435 & .483 & 541 & 525 & .615 \\
\hline PLE-75B & 313 & 382 & AS1 & 515 & .695 \\
\hline 74876 & 824 & 867 & 806 & 2220 & 1450 \\
\hline PQ16B & 89 & 642 & 669 & 1220 & 1.080 \\
\hline PLERTA & .067 & 368 & .690 & .716 & .844 \\
\hline PLE-T7B & 536 & .626 & .658 & .759 & 870 \\
\hline$P+3 \times 18$ & 865 & 934 & 1.050 & 6130 & 1250 \\
\hline PERT & 398 & 462 & 557 & 615 & 737 \\
\hline PLB-79A & .430 & .499 & 546 & .610 & .669 \\
\hline PDE-7918 & 308 & .324 & .371 & .493 & .658 \\
\hline
\end{tabular}

Figure 1 shows the graph obtained by plotting base 10 $\log (B E R)$ versus time for the sixteen disks in cell 1 . This is the graphical display of the data reported in Table 2.

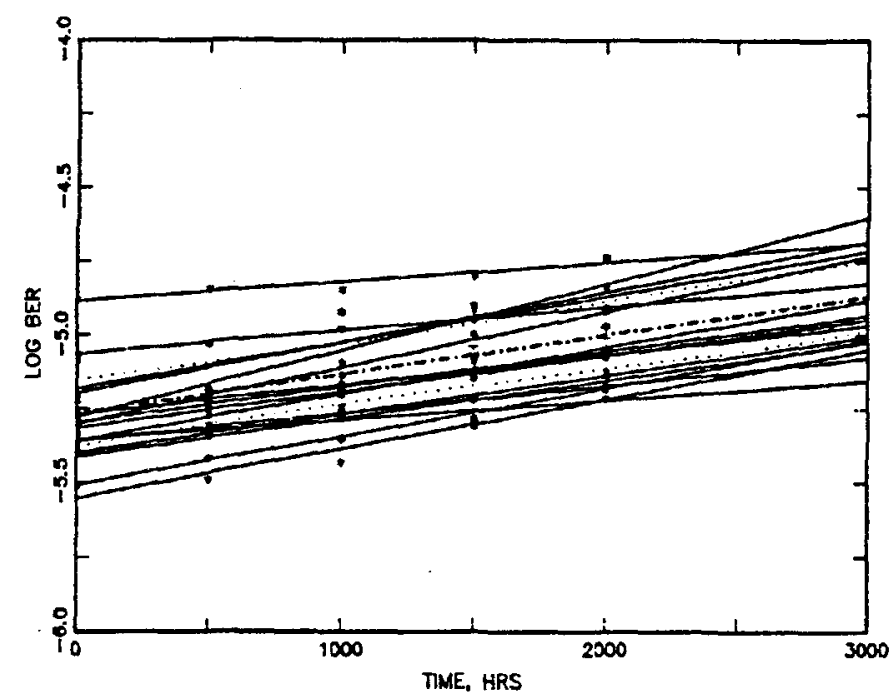

Figure 1: $\log (\mathrm{BER})$ vs time exposed to $80^{\circ} \mathrm{C} / 85 \% \mathrm{RH}$

The definition used for End-of-Life was a BER of $5 \times 10^{-4}$. This value is a very conservative estimate of the minimum level at which the Reed-Solomon ECC would be un able to correct errors. The occurrence of any new spared sector would also have signaled EOL.
As no such occurrences were observed, all lifetimes were based on the maximum likelihood estimated time to reach the BER of $5 \times 10^{-4}$.

Of the 160 sides under accelerated stress, 10 were observed to have zero or negative rates of BER change. This occurred predominantly in the lower temperature cells. These disks were censored from test at the time calculated for the longest surviving disk which showed failure within that cell.

At this point, the beginning of life, degradation rate, degradation pathway and end-of-life was known for each individual disk. The calculated time, in years, to reach EOL was used to determine the distribution of failures within each cell. The distribution functions most likely fitting a lifetime estimate of these disks included Weibull, lognormal, normal and exponential. Each was evaluated and, as expected, the lognormal distribution was found to offer the best fit. The life expectancy data for cell 1, plotted on a lognormal scale, is shown in Figure 2 below:

LOGNORMAL PROBABILTY PLOT, N=16

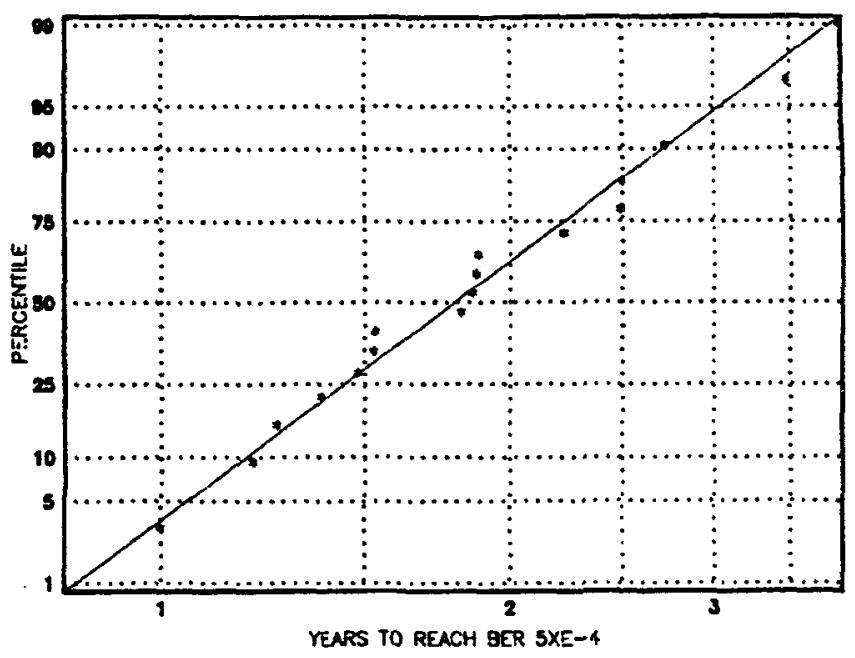

Figure 2: Lognormal plot of Cell $1 \mathrm{EOL}\left(80^{\circ} \mathrm{C} / 85 \% \mathrm{RH}\right)$

It can be seen that an excellent fit was obtained. In the same manner, the shape and scale parameters for lognormal distributions were obtained from each of the experimental cells. The experimentally determined scale parameters (time for $50 \%$ failure for lognormal distributions) for each cell were used to solve the acceleration model.

The scale values thus obtained for each cell along with the cell stress parameters of temperature and relative humidity were regressed to estimate the coefficients of the Eyring model. The MLE regression technique was used throughout. These coefficients were determined to be; $\mathrm{A}=-27.333 \mathrm{yr}^{1}$, 
$\mathrm{E}=.926 \mathrm{eV}$, and $\mathrm{C}=-.063 \mathrm{~T}$. The value of $\mathrm{E}$ closely matches that of $.96 \mathrm{eV}$ found in previous work involving temperature modeling only [6].

Using this model, a scale parameter (time for $50 \%$ failure) may be calculated for any temperature and relative humidity desired. The calculated scale parameter may then be ratioed to the experimentally determined scale parameters for each cell. In this manner, an "Acceleration Factor" (A.F.) may be calculated for each accelerated test cell.

$$
\text { A. F. }=\frac{\text { scale (usage condition) }}{\text { scale (accelerated condition) }}
$$

It bears mentioning that these acceleration factors are relative to a specified usage condition, for a specified degradation mechanism, and only on the particular disk construction evaluated. A table of the acceleration factors for each cell in this model, relative to the "worst case" of $30^{\circ} \mathrm{C} / 90$ \%RH is shown below:

Table 3: Scale and acceleration factors by Cell

$\begin{array}{cccccc}\text { Cell * } & \begin{array}{c}\text { Scale } \\ \text { Factor }\end{array} & \begin{array}{c}\text { Accel } \\ \text { Factor }\end{array} & \text { Cell * } & \begin{array}{c}\text { Scale } \\ \text { Factor }\end{array} & \begin{array}{c}\text { Accel } \\ \text { Factor }\end{array} \\ 1 & 3.76 & 100 & 6 & 8.66 & 43.4 \\ 2 & 4.63 & 81.2 & 7 & 10.7 & 35.1 \\ 3 & 5.70 & 65.9 & 8 & 21.0 & 17.9 \\ 4 & 7.02 & 53.5 & 9 & 32.6 & 11.5 \\ 5 & 20.7 & 18.1 & 10 & 53.7 & 6.99\end{array}$

These acceleration factors are shown graphically as a function of both temperature and relative humidity in figure 3. Both the effect of relative humidity at a given temperature as well as the temperature effect at a given relative humidity may be seen.

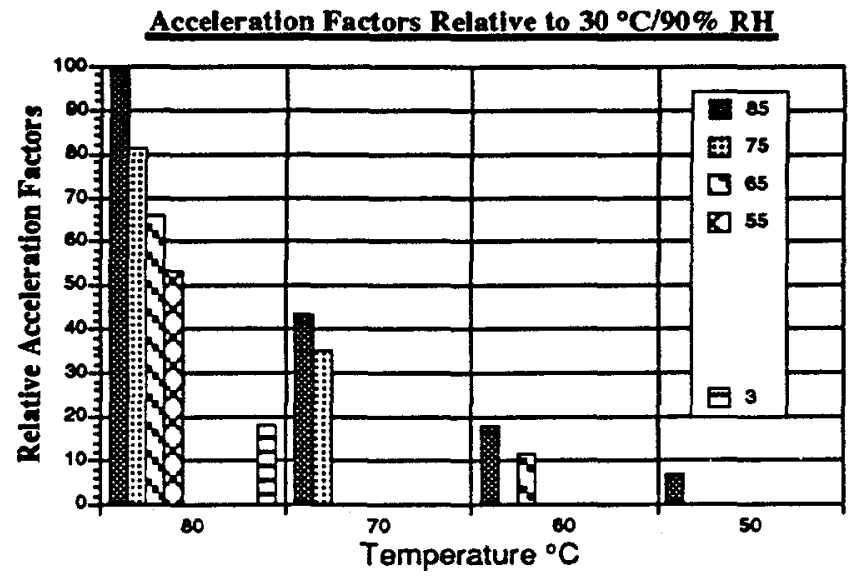

Figure 3: Acceleration Factors by Temp. ${ }^{\circ} \mathrm{C}$ and \%RH
The final test for establishing confidence in this model was obtained by multiplying the relative acceleration factors by the failure times in each cell. This normalized all of the data to the $30^{\circ} \mathrm{C} / 90 \% \mathrm{RH}$ condition. When this combined data was replotted on a common lognormal scale, the fit was again shown to be very satisfactory. This graph is shown in figure 4 below.

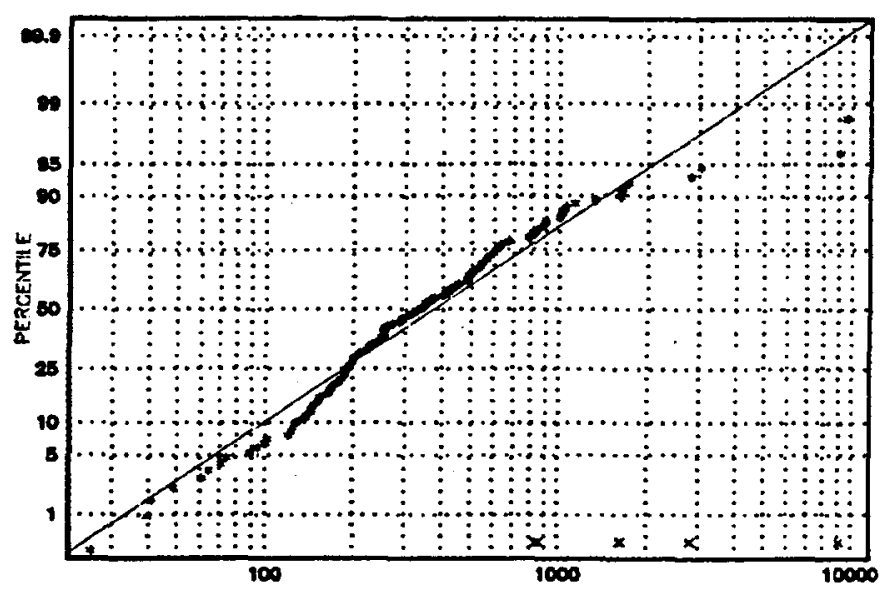

Figure 4: Combined data normalized to $30^{\circ} \mathrm{C} / 90 \% \mathrm{RH}$

The lognormal reliability function may be expressed as:

$$
R(t)=\Phi\{[\log (t)-\mu /\} \sigma
$$

Where $\Phi$ is the standard normal cumulative distribution function. The combined lognormal distribution of the normalized data produced a $\mathfrak{t}(50)=376$ years, mu (log mean $)=5.9287$ and a sigma (log standard deviation) value of 1.0421. The likelihood surface for this data is shown in Figure 5:

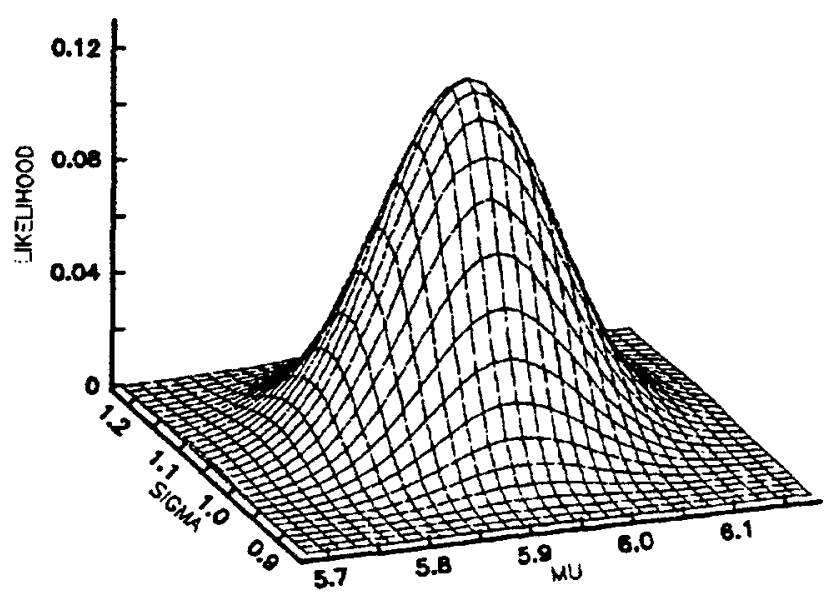

Figure 5: Likelihood surface for lognormal distribution 
The survival function, $R(t)$, can be used to illustrate the population fraction that survives until any specified time $(t)$. Figure 6 shows this function for the data normalized to $30^{\circ} \mathrm{C} /$ $90 \%$ RH. The dotted lines show the $95 \%$ confidence intervals based on asymptotic normal approximation.

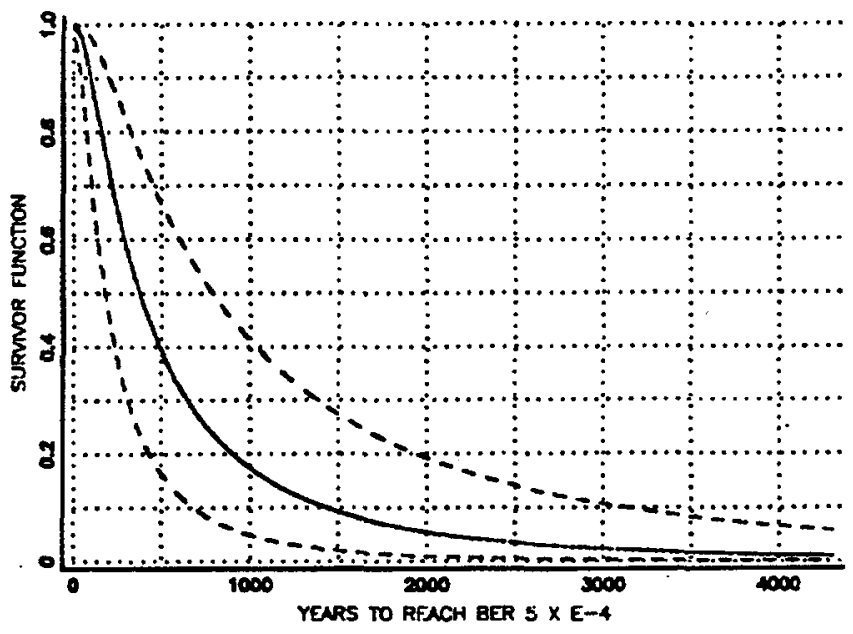

Figure 6: Survival Function for $30^{\circ} \mathrm{C} / 90 \% \mathrm{RH}$

An expansion of the area containing the .95 to 1.0 survival fraction is shown in Figure 7 below:

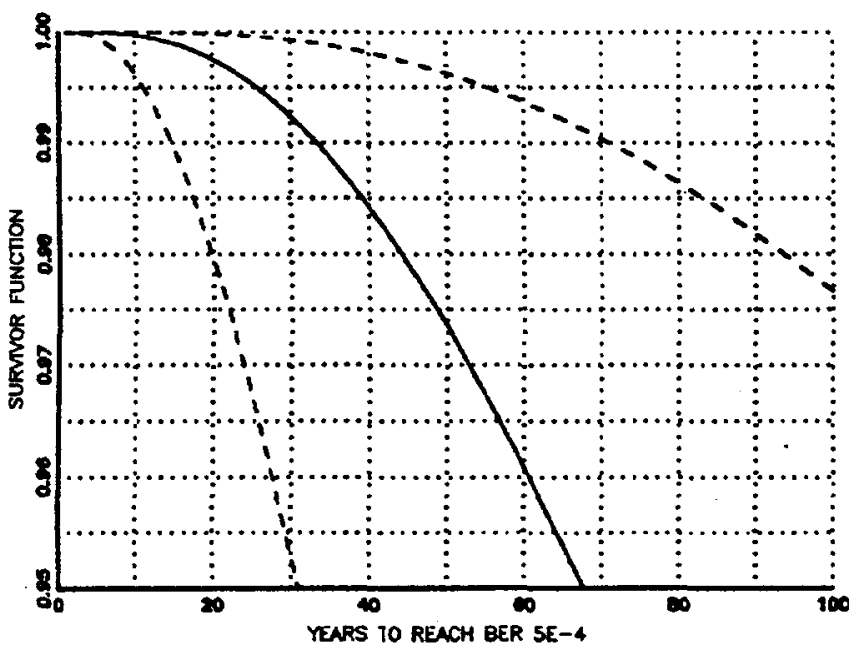

Figure 7: Expanded Area of Survival Function

This figure may be interpreted as indicating that, with $97.5 \%$ confidence, $95 \%$ of $3 \mathrm{M} \mathrm{M}-\mathrm{O}$ media will require at least 30 years to reach a BER of $5 \times \mathrm{E}-4$. Also, with the same confidence, $99.5 \%$ of the product will survive 10 years.

The $30{ }^{\circ} \mathrm{C} / 90 \% \mathrm{RH}$ condition has been historically the point considered as the worst case storage condition. This is likely based on the allowed storage restriction of no condensation and a maximum wet bulb of $29^{\circ} \mathrm{C}$. This combination produces the maximum relative humidity at $30^{\circ} \mathrm{C}$ and a $1{ }^{\circ} \mathrm{C}$ wet bulb depression at that temperature indicates approximately $90 \% \mathrm{RH}$. Okazaki uses a more realistic condition of $20^{\circ} \mathrm{C} / 65 \% \mathrm{RH}$ for his usage condition [7]. This "normal office environment" was used to calculate the 3M M-O media lifetime shown in Figure 8 below:

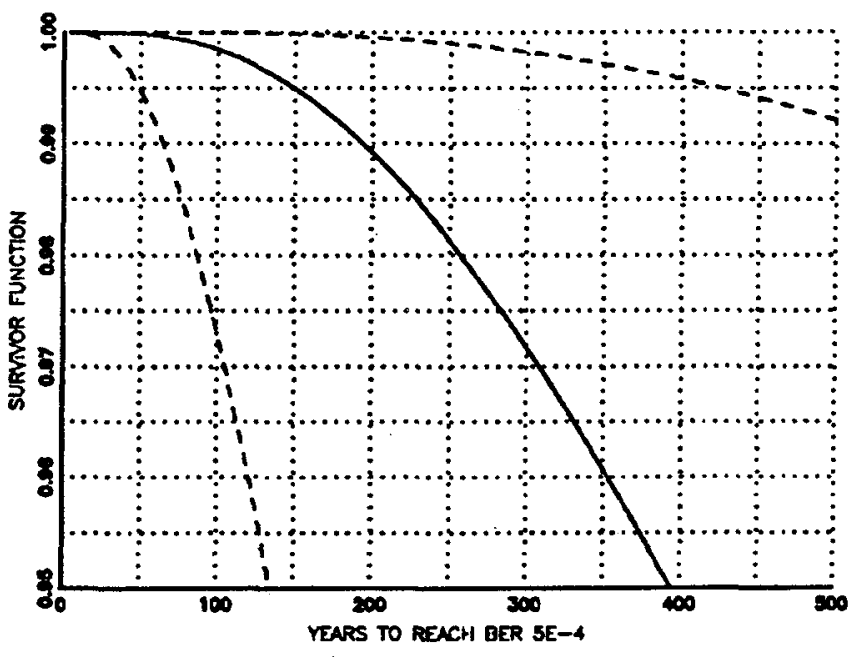

Figure 8: Survival Function for $20^{\circ} \mathrm{C} / 65 \% \mathrm{RH}$

Use of these conditions indicate that, again using $97.5 \%$ confidence, $95 \%$ of the media tested should last approximately 130 years and $99.5 \%$ would require greater than 50 years to reach a BER of $5 \times 10-4$.

Archivist might argue that this is a little cool and damp for typical storage conditions. Their interest might be at a condition of $25^{\circ} \mathrm{C} / 50 \% \mathrm{RH}$. As the model developed above can produce lifetime estimates at any combination of temperature and \%RH, it should be no problem calculating life expectancies at these or other similar points of interest. This is demonstrated by Figure 9 below.

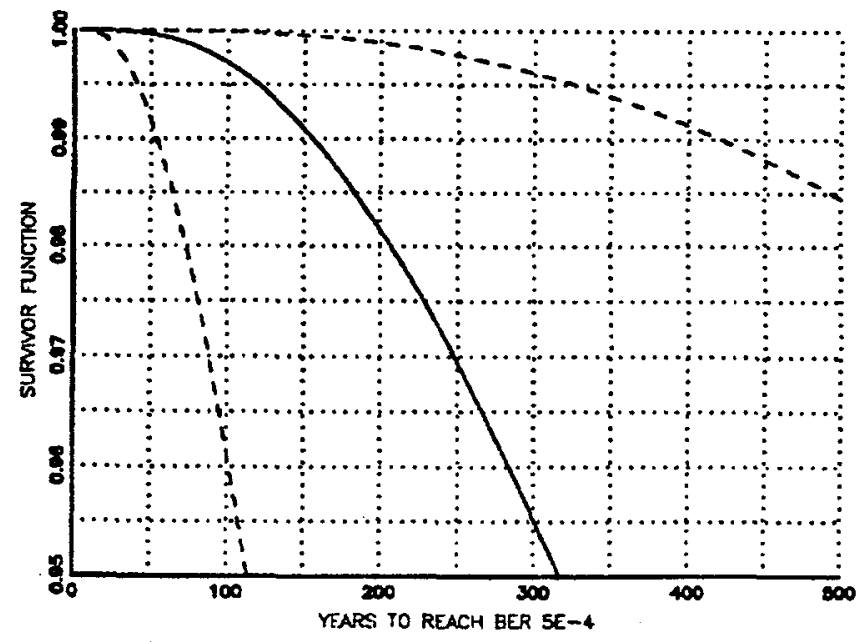

Figure 9: Survival Function for $25^{\circ} \mathrm{C} / 50 \% \mathrm{RH}$ 


\section{CONCLUSIONS}

The Eyring model and the lognormal distribution are common and well accepted expressions in the field of accelerated life expectancy estimation. The combination of these two expressions were shown here to provide an excellent model for the data collected on the $3 \mathrm{M}$ media used in this study. In addition, these life expectancy estimations were further refined by using the maximum likelihood estimate rather than the least squares estimate for regressions and by using the asymptotic normal approximation for confidence intervals.

The total package of analytical techniques allows one to make life estimates at various realistic temperatures and relative humidities. The confidence intervals and percent compliance are also readily obtained. Archivists may now obtain life expectancies at their true storage conditions rather than at hypothetical or experimentally convient conditions.

The calculations show that, at the commonly accepted "worst case" storage conditions of $30^{\circ} \mathrm{C} / 90 \% \mathrm{RH}$, the best estimate indicates $95 \%$ of $3 \mathrm{MM}$ M-O media may be expected to last approximately 68 years without loss of data. It also indicates that, with 97.5 percent confidence, 95 percent of this media will survive at least 30 years. Storage at a more realistic archival condition of $25^{\circ} \mathrm{C} / 50 \%$ humidity extends the best estimate for $95 \%$ survival to greater than 300 years and at least 100 years for the lower side of the 97.5 percent confidence level.

It is impossible to predict the life of any individual disk, as the exact conditions to which it will be exposed are unknown. From this data analysis, however, it appears safe to assume that one may confidently store data on $3 \mathrm{M} \mathrm{M}-\mathrm{O}$ media for time frames on the order of decades without the loss of data. It has been shown that the media behaves in a predictable manner according the traditional accelerated and service life testing techniques. Extension of these conclusions to other media types may not be made without experimental confirmation.

The evolution of $\mathrm{M}-\mathrm{O}$ products and processes have continued. Those of good manufacture are likely to continually improve in quality and reliability [8]. The testing methodology used here also can be improved. Optimizing sample sizes and distribution of samples among the various stress conditions would further enhance the precision of the method. The basic approach of an Eyring model, lognormal distribution, maximum likelihood regression and asymptotic normal approximations should be key elements for this type of accel- erated life estimation. Efforts are underway to produce standardized methodology that that will allow the archivists to obtain life estimates at conditions that are realistically useful. Results produced from such methodology should allow direct comparison of different media, or types of media so that meaningful decisions may be made regarding the most appropriate option for data storage.

\section{REFERENCES}

[1] S. Glasstone, K. J. Laidler, and H. E. Eyring, The Theory of Rate Processes, McGraw-Hill, New York, 1941

[2] P. A. Tobias and D. Trindade, Applied Reliability, Van Norstrand Reinhold, New York, 1986, p. 89

[3] W. Weibull, "A Statistical Distribution Function of Wide Applicability", J. Appl. Mechan. 18, 1951, pp. 293 297

[4] D. Kececioglu, Reliability Engineering Handbook, Prentice-Hall, New Jersey, 1991, p. 425

[5] W.P. Murray, "Advancements in Reliability Testing of Magneto-Optic Systems", J. Magn. Soc. Jpn., 15, Supplement No. S1, 1991, pp. 357-360

\section{[6] Ibid}

[7]H. Okazaki, et al., Proceedings, SPIE Vol 1078, Optical Data Storage Topical Meeting, 1989, pp 51-57

[8] J. S. Wong, et al., "Testing of Commercial Optical Media", IEEE Transactions on Instrumentation and Measurement., 41, No 2, 1992, pp 304 - 307 